\title{
IDEAL RECTIFIER BRIDGE CONVERTING THE HARVESTED ENERGY OF VIBRATIONS INTO ELECTRIC ENERGY TO POWER AN MR DAMPER
}

\author{
Bogdan SAPINSKK", Łukasz JASTRZĘBSKI", Arkadiusz KOZIEL ${ }^{* * * *}$ \\ *Faculty of Mechanical Engineering and Robotics, Department of Process Control, AGH University of Science and Technology, \\ al. Mickiewicza 30, 30-059 Kraków, Poland
}

deep@agh.edu.pl, lukasz.jastrzebski83@gmail.com, arkadiuszkoziel93@gmail.com

received 3 November 2020, revised 19 January 2021, accepted 22 January 2020

\begin{abstract}
The newly developed ideal rectifier bridge equipped with four N-type MOSFETs and two rail-to-rail operational amplifiers is a part of a typical energy harvesting conditioning circuit responsible for the rectification stage in the system of converting the energy harvested from vibrations into electrical energy to power the MR damper. The only energy loss in the bridge is caused by the voltage loss in transistors' channels. The first sections of the work summarises the structural design of the bridge, the simulation procedure under the RL load and by sine voltage inputs with predetermined frequency and amplitude range, and benchmarks the results against those obtained for the conventional bridge based on Schottky diodes. In the second section, the PCB prototype of the bridge is analysed, and measurement data are compiled. The third section reports on the laboratory testing of the developed bridge converting the harvested energy in an MR damper-based vibration reduction system.
\end{abstract}

Key words: Energy Harvesting, Rectifier Bridge, Voltage, Current, MR Damper

\section{INTRODUCTION}

Energy harvesting vibration reduction systems with MR dampers have attracted a great deal of attention recently. Their main advantage is that they are able to effectively reduce the vibration amplitude of the sprung mass at near-resonance frequency using the energy harvested from vibrations. Such systems provided in e.g. automobiles and railway vehicles (Sung and Choi 2008, Wang and Liao 2009a, Wang and Liao 2009b) utilize ambient vibrations to excite the harvester harmonically, thereby producing an alternating voltage or $\mathrm{AC}$ power. The $\mathrm{AC}$ power has to be conditioned before it can be effectively used with any electronics or storage elements requiring DC power (Safei et al. 2019). A typical energy harvesting conditioning circuit has two major components: the rectification (AC/DC conversion) and regulation stage (DC/DC conversion). The simplest energy harvesting circuit is the full-wave rectifier (converting $A C$ signals into all positive voltage) integrated with a smoothing capacitor (converting the signals to $D C$ ).

As demonstrated in Sapiński (2011), the energy harvesting vibration reduction system with an MR damper has three separate components: an electromagnetic harvester, an MR damper and an energy conditioning circuitry. Similar three components can be distinguished in the energy harvesting MR damper reported in Sapiński (2014). It appears that in both cases, the system is able to adjust itself to structural vibrations, however, in the limited range of the harvester output voltages. The constraint imposed by the limited range of the harvester output voltage is the consequence of the predetermined range of MR damper piston velocity (amplitude and frequency of vibrations). Assuming that the har- vester and MR damper parameters are known beforehand, of particular interest is the AC/DC conversion of the harvester output voltage such that the power loss should be as low as possible.

In their former studies, the authors used the rectifier bridge incorporating of the Graetz bridge based on Schottky diodes. Those diodes are widely used in automotive power systems as protection from the effects of reverse battery conditions and of other automotive electrical transients.

When analysing literature on the subject, the authors' attention has been drawn to original solutions concerning rectifier bridges, which are described in many research reports. For example, Balato et al. (2017) demonstrated an H-bridge Graetz rectifier with a smoothing capacitor to convert $\mathrm{AC}$ current generated in resonant electromagnetic vibration harvester into $\mathrm{DC}$ current supplying resistance load. Snamina and Orkisz (2014) proposed an $\mathrm{H}$-bridge Graetz rectifier, enabling the accumulation of energy recovered from vibrations in 2DOF mechanical system in largecapacity capacitors. Grzybek and Micek (2017) described an H-bridge Graetz that was applied in the mechanical system with a self-powered vibration sensor, including piezoelectric material. It should be noted that the rectifier bridges presented in those works concern silicon diodes. The forward voltage drop of the Schottky diodes, however, results in significant power loss at high currents and enhances the need for thermal management using heatsinks and a larger PCB space. For this reason, the authors undertook to develop a new rectifier bridge to eliminate the aforementioned drawbacks so that it should be applicable in MR damper-based vibration reduction systems and recommended the use of N-type MOSFETs in place of Schottky diodes (Selevaraj 2019). 
It is worthwhile to mention the solution by Maiorca et al. (2013) proposed to use of a diode-less mechanical $\mathrm{H}$-bridge rectifier, in which there are no voltage drops in the forward direction. However, this solution is problematic due to the mechanical strength of the rectifier elements and the breakdown that may occur at the time of switching. The solution most similar to the one discussed in this work was proposed by Chytil (2014) where the ideal rectifier bridge was based on a commercial chip that controls four transistors. Also, this solution is not possible to use in the application described in Sapiński (2011) due to the too high voltage (9 V) required for the proper operation of the $\mathrm{H}$-bridge rectifier.

The present paper is organized as follows. Section 2 summarises the design structure and operating principle of the ideal rectifier bridge. Section 3 benchmarks the simulation results of the bridge under the RL load and by sine voltage inputs with predetermined frequency and amplitude range against those obtained for the Graetz bridge based on Schottky diodes. Section 4 describes PCB of the bridge. Section 5 collates the measurement data obtained for the two bridge types and discusses the experimental results in relation to simulation data. Section 6 is focused on the laboratory testing of the developed ideal bridge employed for converting the energy harvested from vibrations into electric energy used to power the MR damper. Finally, the main conclusions are presented in Section 7.

\section{DESIGN STRUCTURE AND OPERATING PRINCIPLE}

The objectives underlying the bridge design are closely associated with parameters of the prototype electromagnetic harvester device (Sapiński 2010) and of the Lord RD-8040-1 MR damper (Lord Corporation 2020) and their application in vibration reduction systems with energy harvesting capability. As shown in Fig. 1, the bridge is positioned between the harvester coil and the control coil of the MR damper. The input to the bridge is a voltage signal induced in the harvester coil (proportional to vibration velocity), the output signal being the rectified voltage, supplying the control coil of the MR damper.

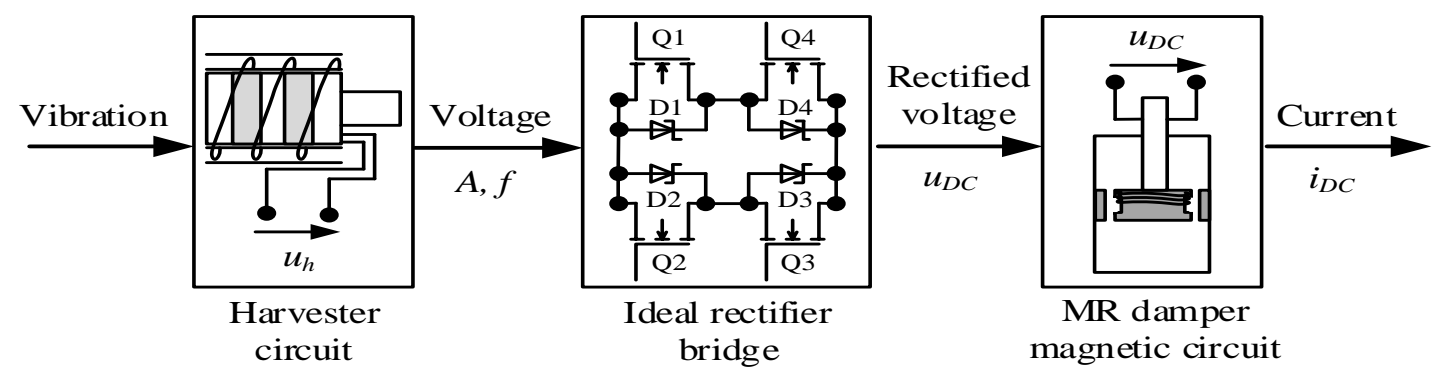

Fig. 1. Schematic diagram of the harvester - ideal rectifier bridge - MR damper system

The underlying assumptions are as follows :

- the maximal amplitude and frequency of voltage induced in the harvester coil should be $A=10 \mathrm{~V}$ and $f=10 \mathrm{~Hz}$,

- resistance and inductance of RD-8040-1 damper coil should be $R_{d}=5 \Omega$ and $L_{d}=100 \mathrm{mH}$,

- the bridge performs with the efficiency approaching $95 \%$ as long as the amplitude of voltage generated by the harvester is of the order of more than $10 \mathrm{mV}$,

- the electric energy required to power the bridge components comes either from the harvester (parasite mode) or from an external local storage device (a Li-ion cell or a battery of capacitors),

- in the conditions of no power supply to the bridge components, the bridge begins to operate as a conventional bridge based on Schottky diodes (with a decidedly lower efficiency).

It is worthwhile to mention that the bridge can be adapted to operate at higher frequency and amplitude of voltage induced in the harvester coil than the levels assumed in the present study.

The diagram of a newly developed bridge (Fig. 2) features three major blocks: the supply block (SB), the control block (CB) and the rectifier block (RB). The BOOST (U1) DC/DC converter in the SB block enhances the input voltage $u_{\text {IN }}$ according to the actual position of the jumper JP1 (available positions: 1, 2, 3). In its position 1 , converter $\mathrm{U} 1$ is supplied with rectified voltage $u_{D C}$, whilst the bridge operates in the parasite mode $U_{I N}=U D C$.

In position 2, converter U1 is supplied with voltage from an external source $U_{E X T}-i$.e. the bridge operates in the external supply mode. Converter U1 elevates the voltage $U_{\text {IN }}$ to $U_{\text {OUT }}=20 \mathrm{~V}$ with respect to the GND reference, when $U_{\mathbb{I N}} \geq 3 \mathrm{~V}$. In position 3 , converter $\mathrm{U} 1$ is discon- nected from the power supply - the bridge operates in the passive mode acting as a conventional Graetz-type rectifying bridge based on Schottky diodes D1-D4.

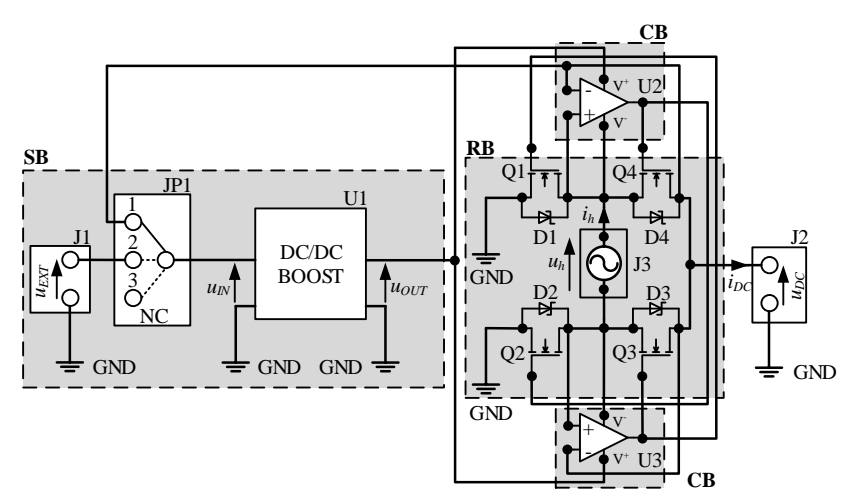

Fig. 2. Schematic diagram of the ideal rectifier bridge

Converter $\mathrm{U} 1$ is requisite to ensure the adequate control of transistors Q1-Q4 whose operation in the linear range is undesirable. Sources of transistors Q3 and Q4 are connected to points of various potential, the potential difference yielding voltage $u_{h}$ (Fig. 2). Consequently, voltage uout must be larger than the sum of $u_{h}$ and the minimal gate-source threshold voltage $U_{G S \min }$ of transistors Q3 and Q4. The block SB is provided with fault and overload protection, as well as $U_{E X T}$ reverse polarity protection and electrostatic discharge protection (ESD). 
Block CB incorporates two operational rail-to-rail amplifiers U2 and $U 3$ operated in the differential configuration with the amplification 10,000 [V/N]. Amplifiers U2 and U3 use the complete span between the negative and positive supply rail for signal conditioning at the input and output. Their function is to amplify the drain-source voltage in transistors Q3 and Q4. The duty cycle of the bridge involves two phases: 1 and 2. At the onset of phase 1, transistors Q1-Q4 remain closed (in off-position), whilst voltage un produces the current flow through diodes D2 and D4. The potential difference between the drain in transistor Q4 and its source is amplified by op-amp U2. The gatesource voltage $U_{G S}$ of transistors $Q 2$ and Q4 is larger than the voltage required for their full opening $\left(U_{G S}>U_{G S \text { min }}\right)$, resulting in immediate current flow $i_{h}$ through transistors Q2 and Q4. Current $i_{D C}$ flows through the two-terminal network $R_{d} L_{d}$ and returns to the harvester coil. The only power loss in the ideal bridge circuit is due to a voltage drop on open channel/drain-source on-state resistances in transistors Q2 and Q4. Throughout phase 1, transistors Q1 and Q3 remain closed, whilst their gate potential is equal to that of their sources. Phase 2, in which transistors Q1 and Q3 are operated and controlled via amplifier U3, proceeds in a similar manner as phase 1.

Block RB is comprised of four N-type MOSFETs (the nominal open channel resistance being $5 \mathrm{~m} \Omega$ ) connected in the $\mathrm{H}$-bridge configuration. Schottky diodes D1-D4 connected in parallel with transistors Q1-Q4 enable the passive mode operation (with no power supply).

\section{SIMULATION PROCEDURE}

Two variants of bridge systems were investigated in the simulation procedure: a conventional rectifier (B1) based on Schottky diodes and an ideal rectifier (B2) incorporating N-type MOSFETs and two rail-to-rail operational amplifiers. The simulation procedure use the LTSpice environment (Analog Devices, 2020). The purpose of the procedure was to determine the extent of power loss in the form of heat released on diodes D1-D4 in bridge B1 and on transistors Q1-Q4 in bridge B2. Key features that have implications for the simulation procedures are as follows:

- the diodes D1-D4 are the type RBR2MM60C (Rohm, 2020), transistors Q1-Q4 are IRFH5007 type (Infineon, 2020);

- the output in each bridge is connected with the control coil of the MR damper modelled as a two-terminal network $R_{d} L_{d}$ (resistance-inductance load), without taking into account the processes in the magnetic circuit;

- the harvester coil is an ideal source of sinusoidally varied voltage with amplitude $A$ and frequency $f$ (its resistance and internal inductance being neglected);

- the capacitor smoothing ripples of voltage $U_{D C}$ in the bridge outlet is neglected;

- in the case of bridge B2, the power supply comes from the battery $U_{E X T}=3.7 \mathrm{~V}$ in the SB block.

Simulation results are illustrated in Figs. 3-6. Apparently, the voltage drop $\left(\left|u_{h}\right|-u_{D C}\right)$ across bridge $B 1$ is about $0.7 \mathrm{~V}$, which is equal to $2 x$ forward voltage drop $U_{F}$ in diodes D1-D4 for the instantaneous current level $i_{D C}$ (Fig. 3a, b). When $A=2 \mathrm{~V}$, the maximal voltage $u_{D C}$ becomes $1.3 \mathrm{~V}$ (Fig. 3a). Negative voltage values UDC (Fig. 3b) registered when voltage $U_{I N}$ falls in the range $\left(-U_{F}, U_{F}\right)$ are attributable to resistance-inductance loading of bridge output (the two-terminal system $R_{d} L_{d}$ ). Voltage drop across bridge B2 is close to zero, whilst $u_{D C} \approx\left|u_{h}\right|$. Power loss in bridge circuit B2 is associated with voltage drop across the drain-source resistances in transistors Q1-Q3 or Q2-
Q4. Voltage drop across the transistors is proportional to their drainsource on state resistance and to current $I_{D C}$. The values of voltage UDC are always positive - block CB with transistors Q1-Q4 is able to effectively dissipate energy stored in the magnetic field of the MR damper control coil.

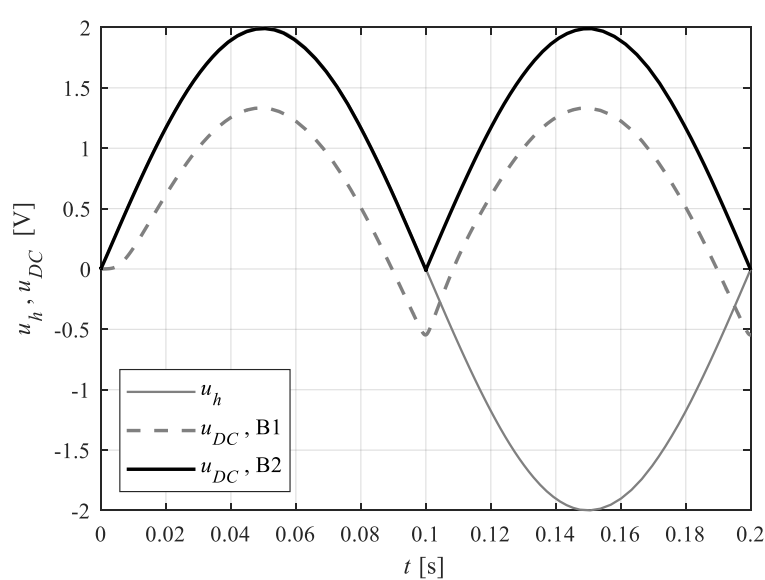

a)

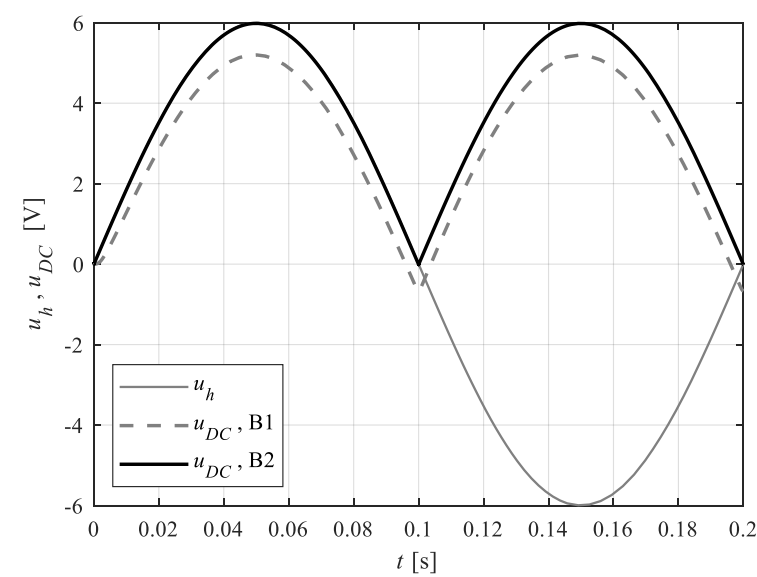

b)

Fig. 3. Time histories of voltage $u_{h}$ and $u_{D C}$ : $f=5 \mathrm{~Hz}$; a) $A=2 \mathrm{~V}$, b) $A=6 \mathrm{~V}$

It appears that within the time period from 0 to $10 \mathrm{~ms}$, when no magnetic field energy is yet accumulated in the damper control coil, the currents $i_{h}$ (Fig. 4a) and $i_{D C}$ (Fig. 5a) in bridge B1 begin to flow when $\left|u_{h}\right|>0.7 \mathrm{~V}$. At the instant the voltage $u_{h}$ reaches zero, the direction of current $i_{n}$ flow is reversed (Figs. 4, 5) while maintaining its absolute value. Furthermore, immediately after the current flow $i_{h}$ is reversed (when $0.1 s<t<0.11 \mathrm{~s}$ ), its absolute value tends to decrease, despite an increase in absolute value of voltage $u_{h}$, which can be attributable to reduced current levels sustained in the circuit by the induction coil. The effect whereby the current flow is sustained by the damper control coil can be also observed for current $i_{D C}$ whose minimal value at each time instant ( $t>0 \mathrm{~s})$ exceeds zero. Lower current levels, in and iDC, in bridge $\mathrm{B} 1$ are associated with the voltage drop across diodes D1D4. The smaller the voltage $U_{D C}$, the smaller the current $i_{D C}$. The average current level $i_{D C}$ for bridge B1 approaches $100 \mathrm{~mA}$ (when $A=2 \mathrm{~V}$, Fig. 5a). For the amplitude $A=6 \mathrm{~V}$ (Fig. $5 \mathrm{~b}$ ), it is approximately equal to $0.5 \mathrm{~A}$. The average current level $i_{D C}$ for bridge $\mathrm{B} 2$ is larger than the average current level registered for bridge $B 1$, its respective values for the amplitudes $A=2 \mathrm{~V}$ and $A=6 \mathrm{~V}$ being $200 \mathrm{~mA}$ and about $700 \mathrm{~mA}$. 


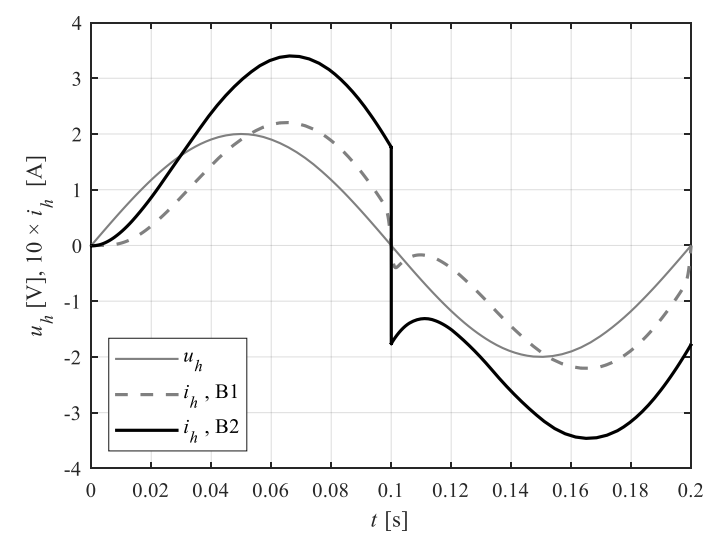

a)

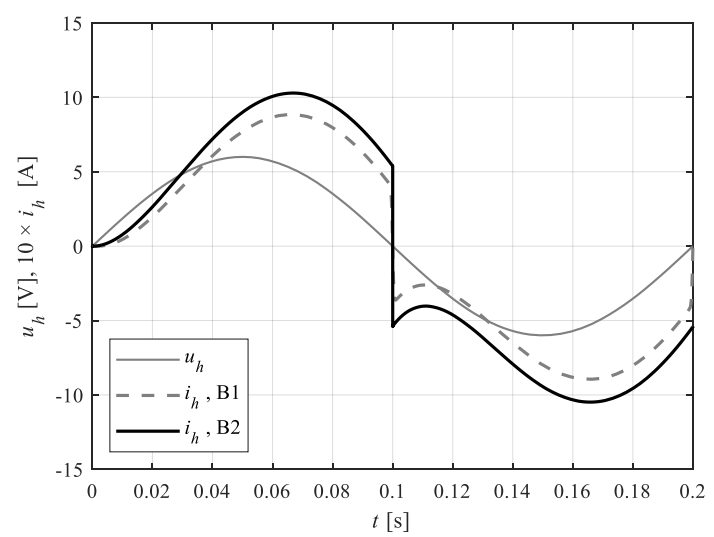

b)

Fig. 4. Time histories of voltage $u_{h}$ and current $i_{h}: f=5 \mathrm{~Hz}$; a) $A=2 \mathrm{~V}$, b) $A=6 \mathrm{~V}$

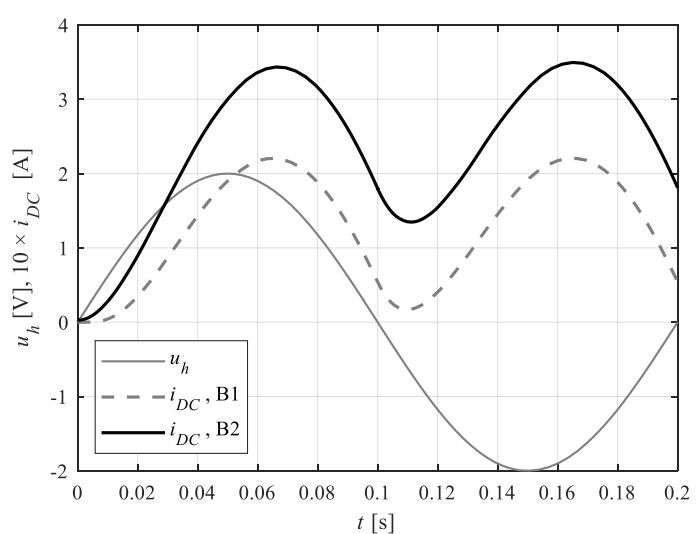

a)

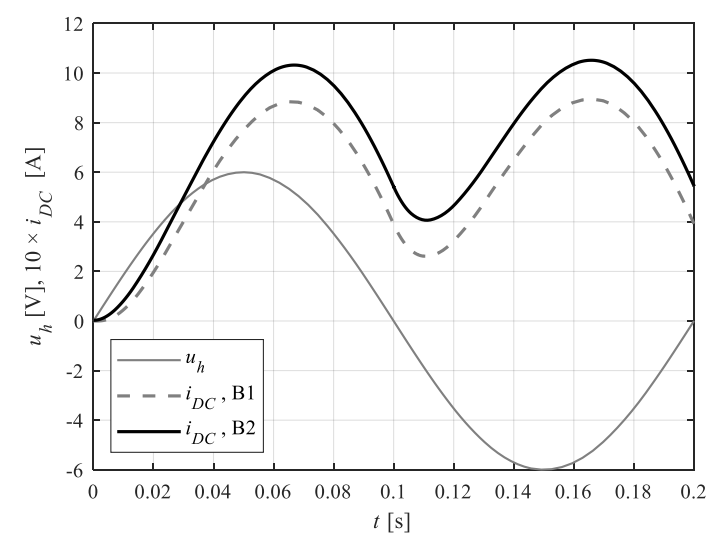

b)

Fig. 5. Time histories of voltage $u_{h}$ and current iDc: $f=5 \mathrm{~Hz}$; a) $A=2 \mathrm{~V}$, b) $A=6 \mathrm{~V}$
Bridge efficiency is derived from the formula:

$\eta=100 \frac{\frac{1}{T} \int_{0}^{t} u_{D C}(t) i_{D C}(t) d t}{\frac{1}{T} \int_{0}^{t} u_{h}(t) i_{h}(t) d t}[\%]$

Plots in Fig. 6 show the bridge efficiency for B1 and B2 in the frequency range $(0.5,10) \mathrm{Hz}$, for amplitudes $A=2 \mathrm{~V}$ and $A=6 \mathrm{~V}$. Apparently, bridge efficiency is improved when the amplitude is 6 $V$. Actually, bridge efficiency $\eta$ is improved at higher amplitude of voltage $u_{n}$ because the voltage $U_{D C}$ supplying the MR damper control coil is equal to voltage $u_{h}$ less the constant voltage value $2 x U_{F}$, being the sum of voltage drops across the bridge components. One has to bear in mind, however, that the actual values of voltage drops $2 x U_{F}$ may differ depending on the type of applied system components (diodes or transistors) and depending on the current level $i_{D C}$ in those elements, whilst they are independent of the input voltage level $u_{h}$. As regards bridge B1, its efficiency decreases with increasing frequency, whilst that of bridge B2 remains nearly constant, exceeding $95 \%$.

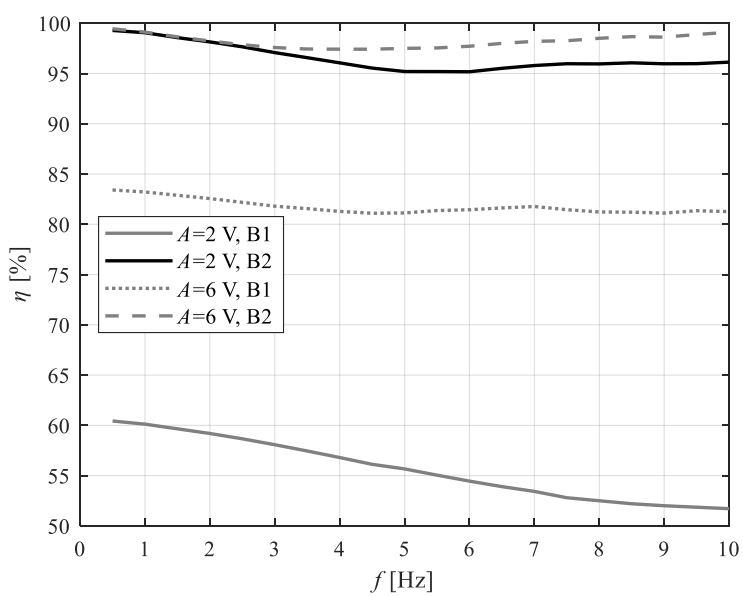

Fig. 6. Efficiency vs frequency

\section{PCB FABRICATION}

The development of PCB design of an ideal bridge (B2) was supported by the KiCad software (KiCad EDA, 2020). All components comprising the $\mathrm{SB}, \mathrm{CB}$ and $\mathrm{RB}$ blocks are implemented on a double-sided laminate. Most components have the surfacemounting device housings. In the double-sided placement configuration, the components are placed in the top and bottom layers, which allows the PCB dimensions to be minimised $(57 \mathrm{~mm} \times 53$ $\mathrm{mm}$ ) and placed on a two-layer copper board. The laminate thickness was $1.6 \mathrm{~mm}$, and the base copper thickness equal to $35 \mu \mathrm{m}$. The copper plating was finished by the HASL (Hot Air Solder Levelling) method; thus, the conductive layer thickness was increased by several $\mu \mathrm{m}$. The path width for signal routing and for supplying the $S B$ and $C B$ clock circuits is $0.2 \mathrm{~mm}$. The paths to handle the block RB and other high current connectors were implemented using the copper planes of paths with the minimal width $0.5 \mathrm{~mm}$. The vias implementing the electric connections between copper layers and effecting the heat release were 0.3 $\mathrm{mm}$ in diameter. The number of vias was increased to handle high-current connections. The view of top and bottom of the bridge PCB is shown in Figs. 7a, b. 


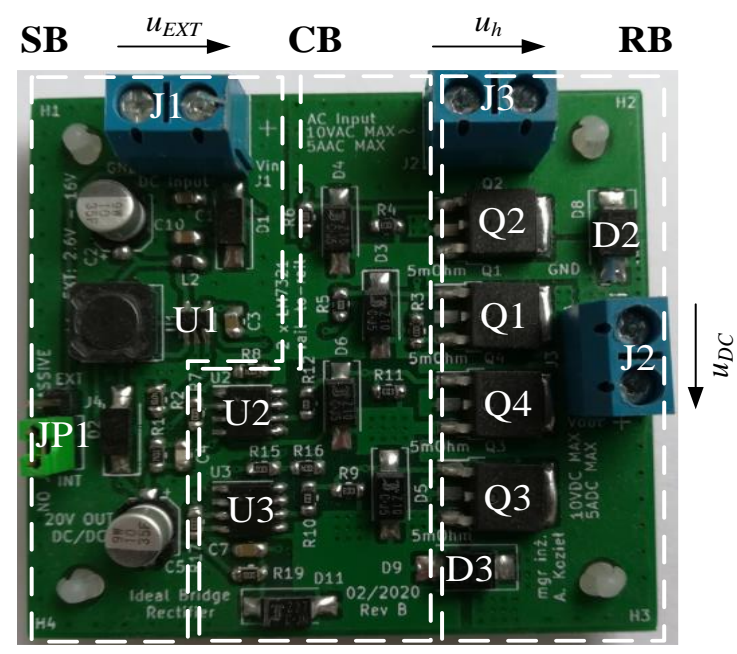

a)

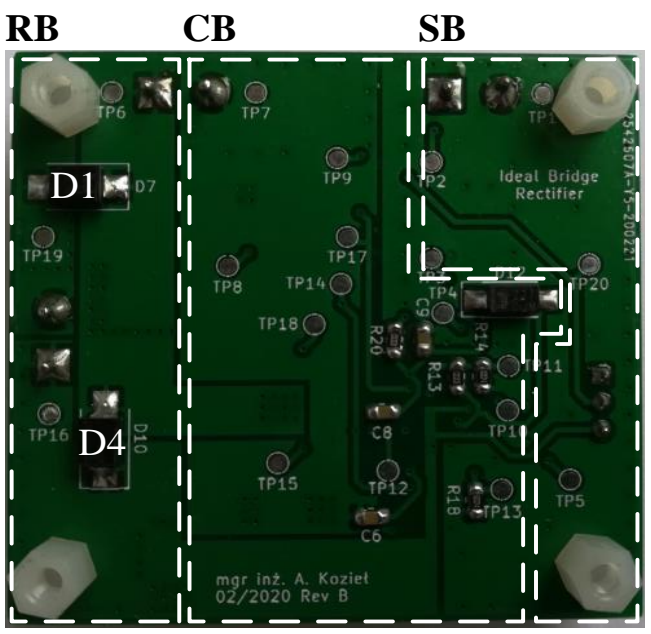

b)

Fig. 7. Ideal rectifier bridge PCB: a) top view, b) bottom view

The PCB surface was subdivided into three respective areas to handle the SB, CB, RB blocks. The connections between regulator U1 and operational amplifiers U2 and U3 were designed to be as short as possible. The decoupling capacitors are placed close to the power supply terminal. Components that release heat (D1-D4 and Q1-Q4) in the RB block are separated from the SB and $\mathrm{CB}$ block circuits. The RB block connections were implemented on the power planes, which enabled the effective heat release to the PCB and to the ambience.

\section{MEASUREMENT PROCEDURE}

Bridge measurements were taken using the circuit shown schematically in Fig. 8, comprising a power amplifier (Sapiński et al. 2012) supplied from the power supply, voltage and current converters, an AD/DA board and a PC supporting the MATLAB/Simulink 2011b. The power amplifier generates alternating voltage fed to the bridge input (corresponding to uh) in accordance with the command signal $u_{\text {con }}$ generated at the AD/DA board output. The amplifier is fitted with integrated converters, enabling the measurements of voltage $u_{n}$ and current in in the harvester coil. Voltage UDC and current iDC in the MR damper control coil are measured with voltage-voltage (u/u) and currentvoltage (i/u) converters based on op-amps AD629 and AD8622.
Registered quantities were duly converted into voltage signals in the range $(+10,-10) \mathrm{V}$, sampled by the $A D / D A$ board at the frequency $1 \mathrm{kHz}$.

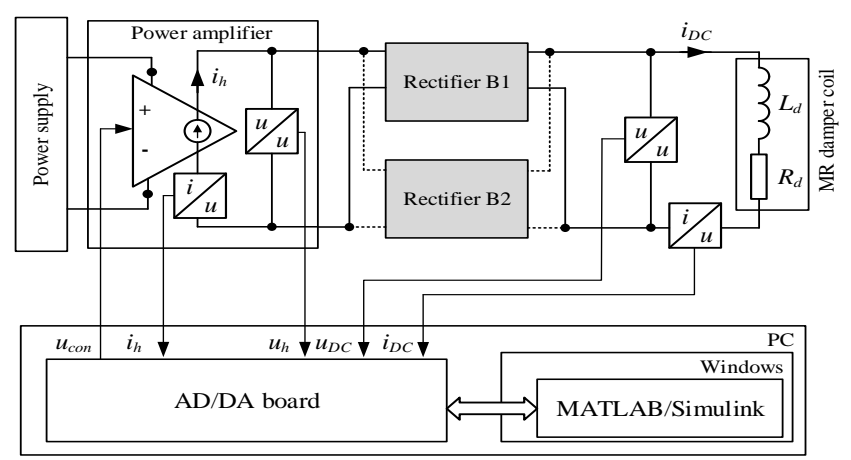

Fig. 8. Diagram of the measurement system

Finally, B2 measurement data are benchmarked against the results obtained for bridge B1 (based on Schottky diodes). One has to bear in mind that the measurements of bridge $\mathrm{B} 2$ were taken using an external power supply (a Li-lon 3.7 V battery) - JP1 in position 2. Plots in Figs. 9-11 show time histories of measured and simulated steadystate voltages $u_{h}, u_{D C}$ and currents $i_{h}, i_{D C}$, Fig. 12 plots efficiency $\eta$ in the function of frequency 1 , derived from formula (1).
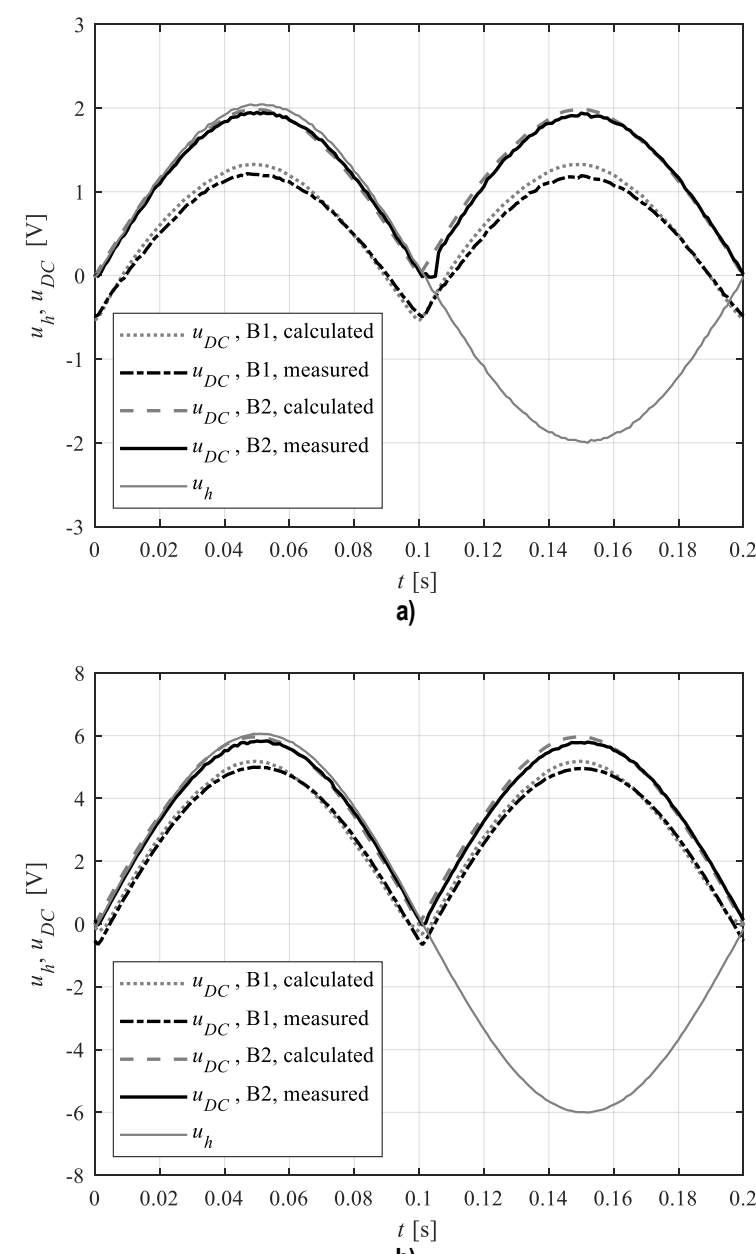

Fig. 9. Time histories of voltage $u_{n}$ and $u_{D C}: f=5 \mathrm{~Hz}$; a) $A=2 \mathrm{~V}$, b) $A=6 \mathrm{~V}$ 


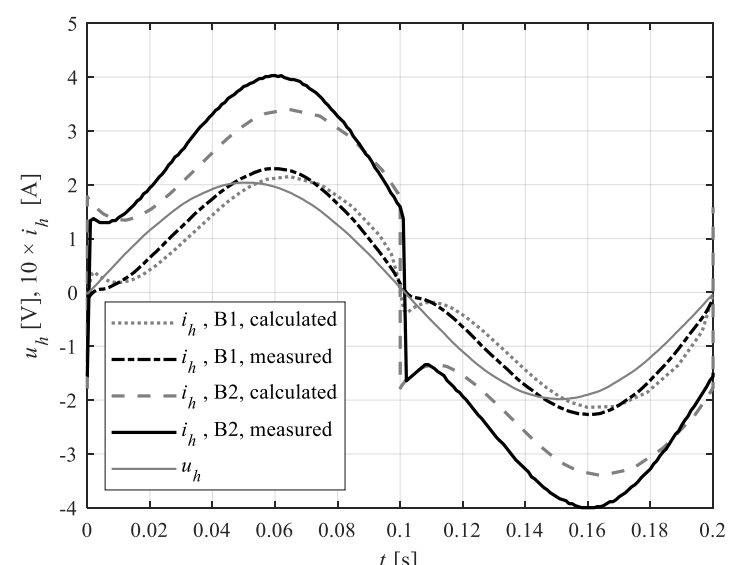

a)

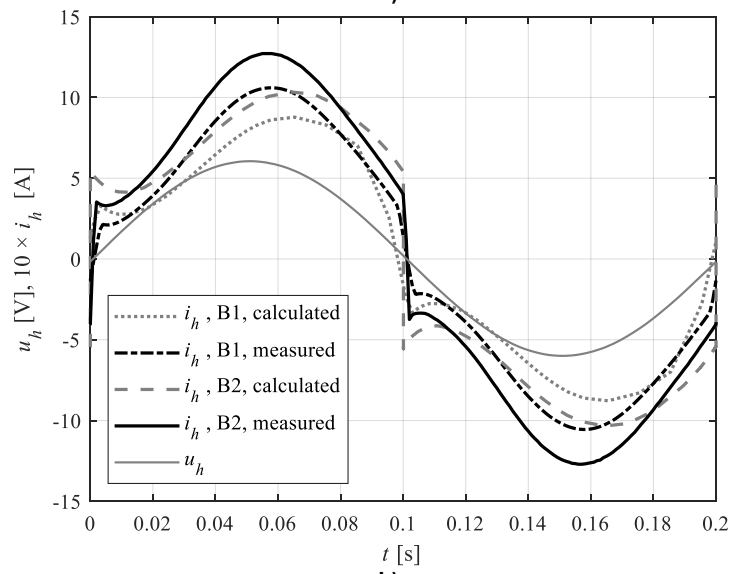

b)

Fig. 10. Time histories of voltage $u_{h}$ and current $i_{h}: f=5 \mathrm{~Hz}$; a) $A=2 \mathrm{~V}$, b) $A=6 \mathrm{~V}$

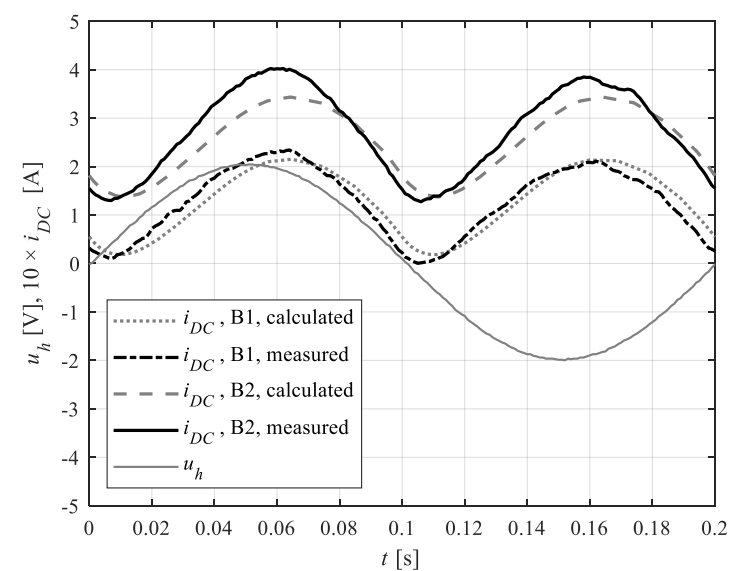

a)

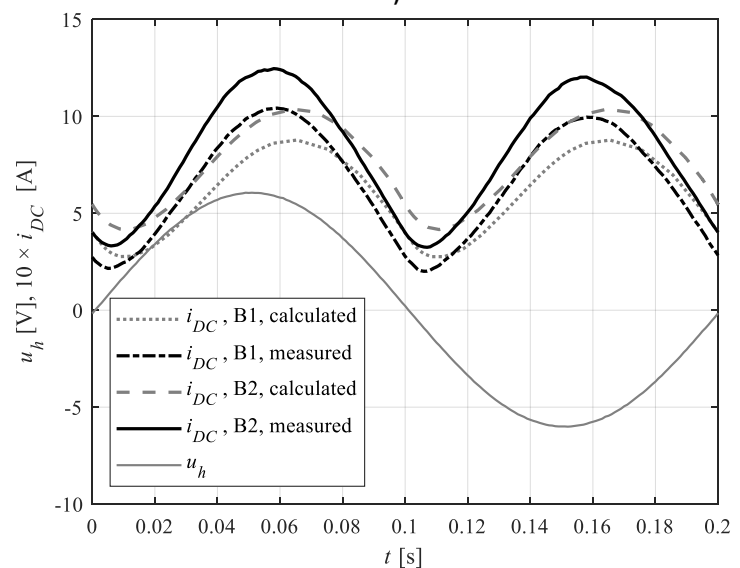

b)

Fig. 11. Time histories of voltage $u_{h}$ and current $i_{D C} ; f=5 \mathrm{~Hz} ;$ a) $A=2 \mathrm{~V}$, b) $A=6 \mathrm{~V}$
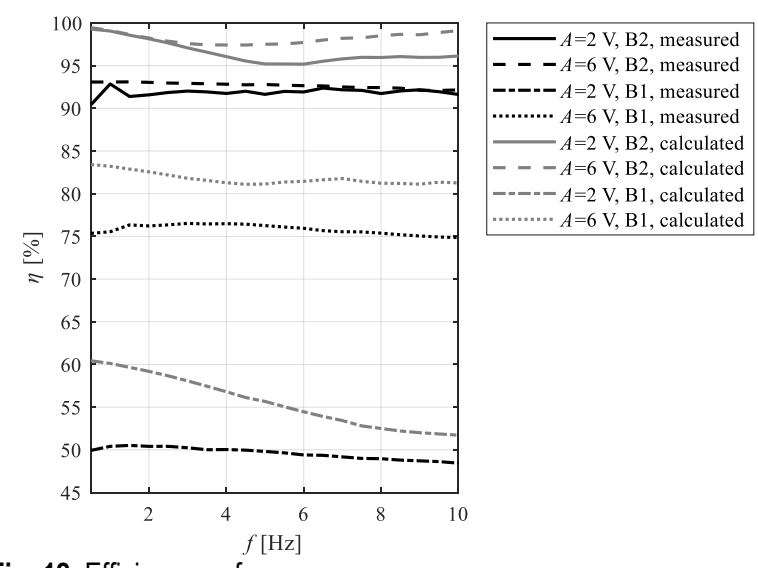

Fig. 12. Efficiency vs frequency

Measured voltages $u_{D C}$ (Fig. 9) are in good agreement with the calculation results. Minor discrepancies are attributable to parasite resistances present in the bridge, not accounted for in the simulation model. The plots of $i_{h}$ and $i_{D C}$ (Figs 10-11) reveal certain discrepancies. The maximal difference between their instantaneous values for $A=6 \mathrm{~V}$ is found to be $0.2 \mathrm{~A}$ and is registered for the two bridges under consideration. Actually, these discrepancies are the result of eddy currents induced in ferromagnetic material from which the MR damper rod is made. The simulation procedure used a solenoid coil where such phenomenon should not occur. The efficiency of bridge B2 obtained experimentally is $92 \%$; for bridge $B 1$, the respective values are: $50 \%$ (for $A=2 \mathrm{~V}$ ) and $75 \%$ (for $A=6 \mathrm{~V}$ ). These are found to be less than the simulated results, mostly due to the occurrence of eddy currents and parasite resistances (Fig. 12).

\section{APPLICATION AND TESTING}

To verify the adequacy of the newly developed bridge B2 design, experimental tests were conducted in the test rig (Sapiński et al. 2010) incorporating the harvester prototype, the RD-8040-1 damper, a passive spring rated at $k=90,000 \mathrm{~N} / \mathrm{m}$ attached to the sprung mass $m=155 \mathrm{~kg}$, a shaker and a measurement system (Fig. 13). The natural frequency of the SDOF system (sprung mass-damper-spring) is $f_{0}=3.8$ $\mathrm{Hz}$. The measurement system comprised a PC, an AD/DA board supporting the MATLAB/Simulink, displacement sensors (S1, S2), force sensors (S3, S4), voltage-voltage (u/u) and current-voltage (i/u) converters. Measurements were taken of the shaker core displacement (input excitation) $z$, sprung mass displacement $x$, force $F_{\text {in }}$ generated by the shaker, damper force $F_{d}$, voltage and current levels in the harvester coil $u_{h}$ and $i_{h}$, voltage and current in the bridge $u_{D C}$ and $i_{h}$ (voltage and current in the MR damper control coil).

The excitations applied in the testing procedure were in the form of displacement inputs $z$ with the amplitude $3.5 \mathrm{~mm}$ and frequency varied from $2 \mathrm{~Hz}$ to $10 \mathrm{~Hz}$, with the step $0.1 \mathrm{~Hz}$. Each test took $30 \mathrm{~s}$ and involved three phases: increasing the amplitude of applied inputs $(0,10) \mathrm{s}$, maintaining the amplitude of applied excitation on the fixed level $(10,20) \mathrm{s}$, decreasing the amplitude of excitation $(20,30) \mathrm{s}$. Figs. 14-16 plot the results of tests done in the time interval $(10,20) \mathrm{s}$, while the amplitude $z$ of the applied excitation remained constant. Figs. 14-15 show the respective time histories of input voltage and current $\left(u_{h}, i_{h}\right)$, output voltage and current $\left(U_{D C}, i_{D C}\right)$ in bridges B1 and B2. Fig. 16 plots the displacement transmissibility coefficient $T_{x z}$ in the function of frequency for the SDOF system governed by formula (2); expressing the efficiency of reduction of the sprung mass vibration amplitude. 
$T_{x z}=\frac{\sqrt{\int_{0}^{T} x(t)^{2} d t}}{\sqrt{\int_{0}^{T} z(t)^{2} d t}}$

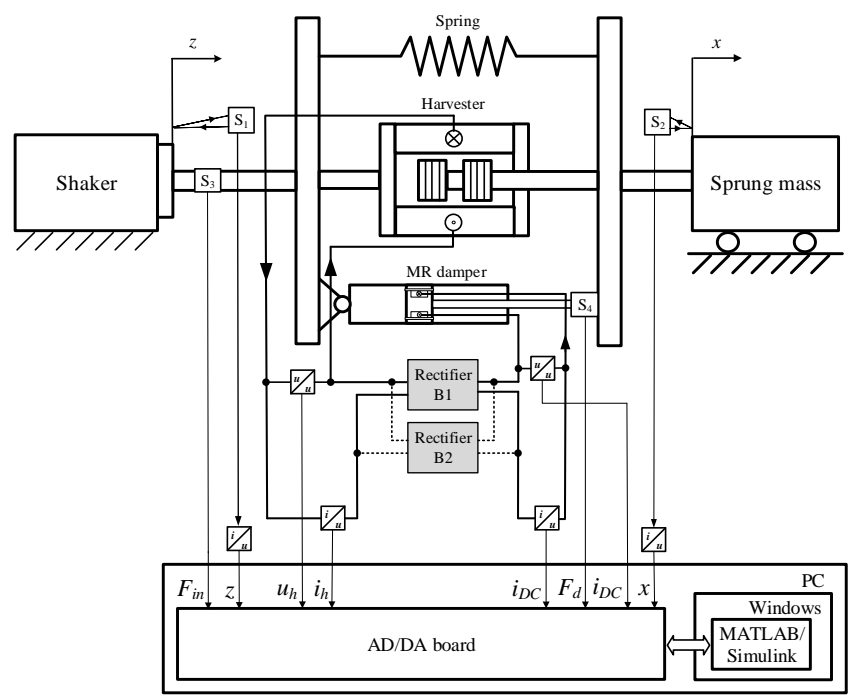

a)

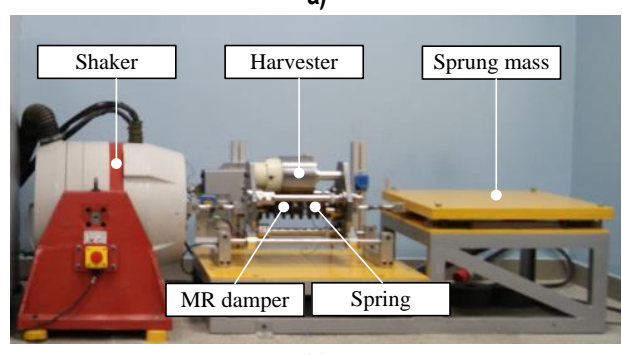

b)

Fig. 13. The test rig: a) schematic diagram, b) general view

It is readily apparent (see Fig. 14) that instantaneous values of voltage in the harvester $u_{h}$ supplying bridge B1 are larger than the voltage supplying bridge $\mathrm{B} 2$ - it appears to be the consequence of a larger relative velocity of magnets motion with respect to the harvester coil, which is associated with a larger amplitude of sprung mass vibrations $x$ (see Fig. 16). The maximal instantaneous values are about $2 \mathrm{~V}$ (B1) and 1.4 V (B2). Despite lower values of voltage $u_{h}$ at the input to $B 2$, currents in in the two bridges are similar in value, not exceeding $200 \mathrm{~mA}$. Moreover, instantaneous values of voltage UDC at the bridge outputs are similar, too (Fig. 15), whilst the only registered discrepancy is the occurrence of negative values of instantaneous voltage, not exceeding $-350 \mathrm{mV}$ in the case of bridge B1. As regards bridge $\mathrm{B} 2$, no negative values of instantaneous voltage $U_{D C}$ are registered, and so, the instantaneous current intensity $I_{D C}$ in the damper control coil reaches a slightly higher value than in $\mathrm{B} 1$, which is indicative of lower loss of energy supplied to the control coil via bridge B2. In consideration of the voltage difference $\left|u_{h}-u_{D}\right|$, it appears that the voltage drop across bridge $\mathrm{B} 1$ is $0.7 \mathrm{~V}$, whilst for bridge $\mathrm{B} 2$, it becomes $20 \mathrm{mV}$. This observation is in good agreement with the results of simulations and measurements, outlined in Sections 4 and 5.

Transmissibility plots $T_{x z}$ in Fig. 16 were derived under the following conditions of power supply to the MR damper control coil (the two-terminal network): no power supply (Case 1), direct supply from the harvester (Case 2), power supply from bridge B1 (Case 3), power supply from bridge B2 (Case 4). Apparently, the amplitude of sprung mass vibration in Case 4 is vastly reduced in the frequency range $(2,6.5) \mathrm{Hz}$ in relation to Case 2 , whilst the maximal value of this coefficient registered at $3.9 \mathrm{~Hz}$ approaches $T_{x z}=1.4$. Its respective values registered in the remaining cases are: $T_{x z}=3.4$ at frequency 3.8 $\mathrm{Hz}$ (Case 1); $T_{x z}=1.6$ at frequency $4.1 \mathrm{~Hz}$ (Case 2); $T_{x z}=1.7$ at frequency $3.5 \mathrm{~Hz}$ (Case 3).

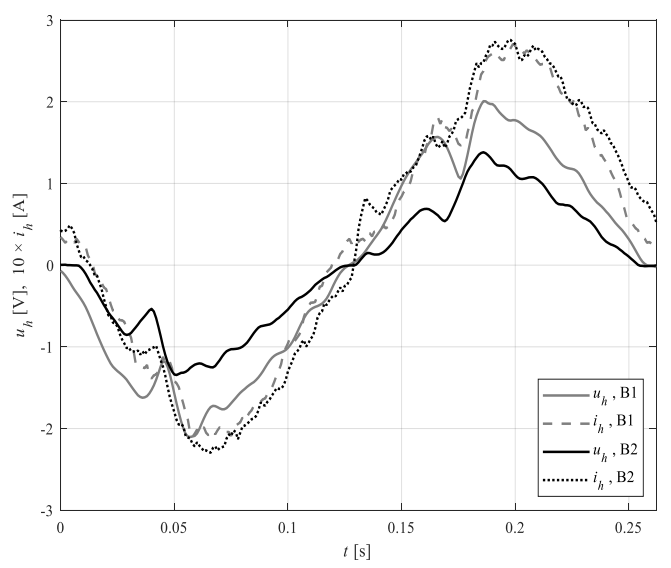

Fig. 14. Time histories of voltage $u_{h}$ and current $i_{h} ; f=3.8 \mathrm{~Hz}$

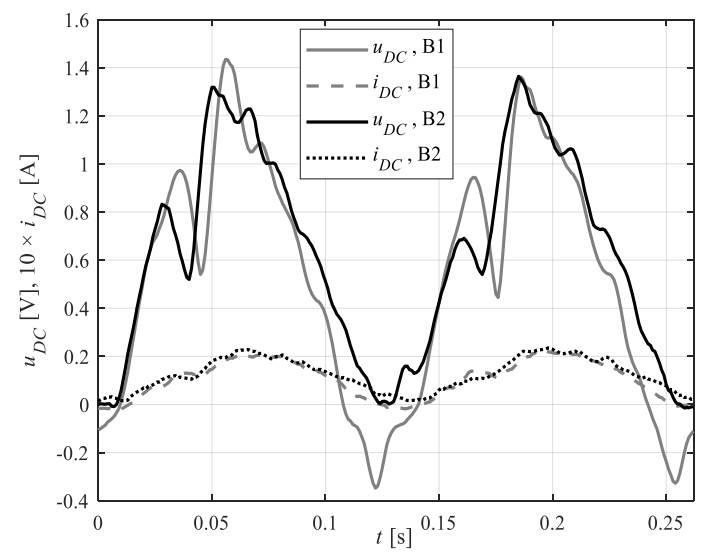

Fig. 15. Time histories of voltage $u_{D C}$ and current $i_{D C} ; f=3.8 \mathrm{~Hz}$

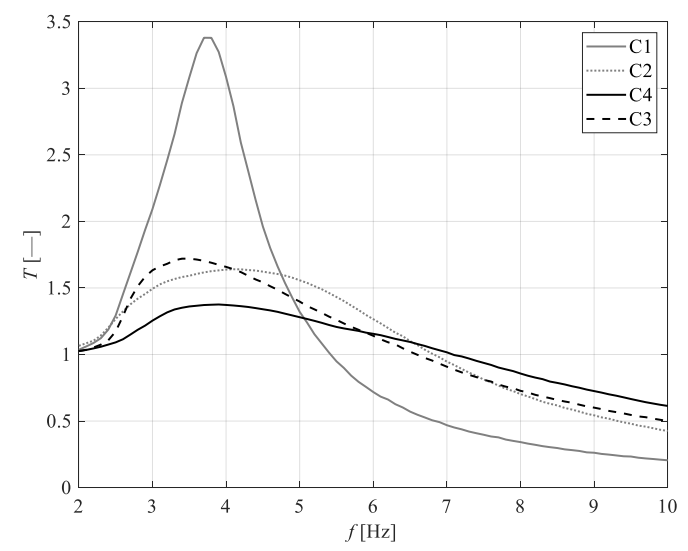

Fig. 16. Transmissibility coefficient $T_{x z}$ vs frequency $f$

Obviously (see the plots in Fig. 1), the application of bridge B2 should be most favourable, as the amplitude of spring mass vibration is thus significantly reduced in the neighbourhood of resonance frequency, whilst the system's behaviour is less favourable at frequencies in excess of $5 \mathrm{~Hz}$ in Cases 2, 3, 4 when compared to Case 1.

This effect can be attributed to the presence of an MR damper dissipating energy placed in between the source of vibration (kin- 
ematic input) and the sprung mass. In such system, when the excitation frequency should exceed $\sqrt{ } 2 f_{0}$, the increase of damping in the system results in increased amplitudes of the sprung mass vibration. Its performance and condition will further deteriorate due to growing amounts of electric energy generated by a harvester at frequencies in excess of $5 \mathrm{~Hz}$. In order to overcome this drawback, it is suggested that additional passive circuits converting the alternating current should be provided in between the harvester coil and the MR damper coil (Jastrzębski and Sapiński, 2017).

\section{SUMMARY}

The main aim of the study was to develop an ideal rectifier bridge to convert the AC signal to positive voltages in an MR damper-based regenerative vibration reduction system. Its structural design and fabrication techniques are outlined, and the results of simulations and laboratory testing are collated. The advantages of the newly developed bridge are demonstrated by benchmarking the measurement results against those obtained for a rectifier bridge based on Schottky diodes, leading us to the following conclusions.

- In the case of bridge B1, the power loss in the harvester coil MR damper control coil circuit is largely due to power loss in Schottky diodes (in the form of heat release). The actual level of power loss is associated with forward voltage and current flowing in diodes. In the case of bridge B2, the power loss is associated with the drain-source on-state resistance of transistors and current flowing through them. Compared to bridge $\mathrm{B} 1$, power loss is significantly reduced, and thus, the efficiency of the bridge (B2) can be improved.

- Efficiency of bridge B1 increases with increasing amplitude of voltage $u_{h}$ and tends to decrease with increasing frequency. Efficiency of bridge B2 is constant; it is unrelated to the amplitude of voltage $u_{h}$ and frequency $f$. For bridge B1, the efficiency becomes $75 \%$, for $\mathrm{B} 2-92 \%$.

- Bridge B2 requires an external source of electric power to supply the electronic circuit for transistor control. This energy may be obtained by harvesting the energy of sprung mass vibration. In the case of no external supply, efficiency of bridge $\mathrm{B} 1$ is similar to that of bridge $\mathrm{B} 2$.

- In the vibration reduction system incorporating bridge B1, the value of transmissibility coefficient $T_{x z}$ will not change by more than $\pm 6 \%$ in relation to the case where the MR damper control coil is supplied directly from the harvester. Apparently, the amplitude of sprung mass vibration is decreased only in the frequency range $(4,7) \mathrm{Hz}$.

- Application of bridge B2 in the vibration reduction system results in a nearly $15 \%$ reduction of the sprung mass vibration amplitude in relation to the case where the bridge is absent. This effect is registered in the frequency range $(2,6.5) \mathrm{Hz}$.

- In each investigated case where the MR damper control coil is supplied from the harvester (with or without the bridge), at frequencies exceeding $5 \mathrm{~Hz}$, the amplitude of sprung mass vibration tends to increase in relation to the case where the coil is not supplied. This is best revealed in systems incorporating bridge $\mathrm{B} 2$, and this drawback can be overcome through the application of passive electric circuits utilising the voltage resonance effect.

Further studies will investigate the electronic systems for conditioning of voltage generated by the harvester, and a dedicated high-efficiency DC/DC converter is planned to be developed.

\section{REFERENCES}

1. Balato M., Costanzo L., Vitelli M. (2017), Resonant electromagnetic vibration harvesters: Determination of the electric circuit parameters and simplified closed-form analysis for the identification of the optimal diode bridge rectifier DC load. International Journal of Electrical Power and Energy Systems 84, 111-123.

2. Chytil J. (2014), Practical realization of ideal diode full-wave rectifiers, Informatics Control Measurement in Economy and Environment Protection, vol.4, no.4, 81-84.

3. Grzybek D., Micek P. (2017), Piezoelectric beam generator based on MFC as a self-powered vibration sensor, Sensors and Actuators A: Physical, 267, 417-423.

4. Jastrzębski Ł., Sapinski B. (2017), Electrical interface for an MR damper-based vibration reduction system with energy harvesting capability. Proceedings of 18 th International Carpathian Control Conference ICCC 2017.

5. Maiorca F., Giusa F., Trigona C., Ando B., Bulsara A. R., Baglio S. (2013), Diode-less mechanical H-bridge rectifier for "zero threshold" vibration energy harvesters, Sensors and Actuators A: Physical, 201, 246-253.

6. Safaei M., Sodano H. A., Steven R Anton S. R. (2019), A review of energy harvesting using piezoelectric materials: state-of-the-art a decade later (2008-2018), Smart Materials and Structures, 28, 113001.

7. Sapiński B. (2010), Vibration power generator for a linear MR damper, Smart Materials and Structures, 19, 105012.

8. Sapiński B. (2011), Experimental study of a self-powered and sensing MR-damper-based vibration control system, Smart Materials and Structures, 20, 105007.

9. Sapiński B. (2014), Energy-harvesting linear MR damper: prototyping and testing, Smart Materials and Structures, 23, 035021.

10. Sapiński B., Jastrzębski Ł., Rosół M. (2012), Power amplifier supporting MR fluid-based actuators, Proceedings of 13th International Carpathian Control Conference ICCC 2012, 612-616.

11. Sapiński B., Snamina J., Jastrzębski Ł., Staśkiewicz A. (2010), Laboratory stand for testing of self-powered vibration reduction systems, Journal of Theoretical and Applied Mechanics, Vol. 49, No. 4.

12. Selevaraj K. (2019), Basics of Ideal Diodes, Texas Instruments, http://www.ti.com/lit/an/slvae57/slvae57.pdf

13. Snamina J., Orkisz P. (2014), Energy Harvesting from Vibrations of a Two-Degree-of-Freedom Mechanical System, Acta Physica Polonica A, vol. 125, no. 4A, 174-178.

14. Sung K. G., Choi S. B. (2008), Effect of an electromagnetically optimized magnetorheological damper on vehicle suspension control performance. Proc. of the Institution Mechanical Engineers Part D Journal of Automobile Engineering.

15. Wang D. H., Liao W. H. (2009a), Semi-active suspension systems for railway vehicles using magnetorheological dampers. Part I: System integration and modelling, Vehicle System Dynamics.

16. Wang D. H., Liao W. H. (2009b), Semi-active suspension systems for railway vehicles using magnetorheological dampers. Part II: Simulation and analysis, Vehicle System Dynamics.

17. Lord Corpotation (2020), MR damper, RD-8048-1, Technical documentation, www.lord.com

18. KiCad EDA (2020), User manual, https://www.kicad-pcb.org/

19. Analog Devices (2020), LTSpice, User manual, https://www.analog.com/

20. Infineon (2020), IRFH5007, Technical documentation, https://www.infineon.com

21. RHOM (2020), RBR2MM60C, Technical documentation, https://www.rohm.com/

This work is supported by AGH University of Science and Technology under research program No. 16.16.130.942. 